\title{
ON THE SUM OF ELEMENT ORDERS OF FINITE ABELIAN GROUPS
}

\author{
BY \\ MARIUS TĂRNĂUCEANU and DAN GREGORIAN FODOR
}

\begin{abstract}
In this note some properties of the sum of element orders of a finite abelian group are studied.

Mathematics Subject Classification 2010: 20D60, $20 \mathrm{D} 15$.

Key words: finite groups, sum of element orders.
\end{abstract}

\section{Introduction}

Let $G$ be a finite group. We define the function $\psi(G)=\sum_{a \in G} o(a)$, where $o$ (a) denotes the order of $a \in G$. The starting point for our discussion is given by the papers $[1,2]$ which investigate the minimum/maximum of $\psi$ on the groups of the same order.

Recall that the function $\psi$ is multiplicative, that is if $G_{1}$ and $G_{2}$ are two finite groups satisfying $\operatorname{gcd}\left(\left|G_{1}\right|,\left|G_{2}\right|\right)=1$, then $\psi\left(G_{1} \times G_{2}\right)=\psi\left(G_{1}\right) \psi\left(G_{2}\right)$. By a standard induction argument, it follows that if $G_{i}, i=1,2, \ldots, k$, are finite groups of coprime orders, then

$$
\psi\left({\underset{i=1}{k}}_{G_{i}}\right)=\prod_{i=1}^{k} \psi\left(G_{i}\right) .
$$

This shows that the study of $\psi(G)$ for finite nilpotent groups $G$ can be reduced to $p$-groups.

In the current note we will focus on the restriction of $\psi$ to the class of finite abelian groups $G$. In this case we are able to give an explicit formula 
for $\psi(G)$. We prove that abelian $p$-groups of a fixed order are determined by this quantity and we conjecture that this happens for arbitrary finite abelian groups. Other interesting properties of the function $\psi$ will be also discussed.

Most of our notation is standard and will not be repeated here. Basic concepts and results on group theory can be found in [3, 4]. For subgroup lattice notions we refer the reader to [5].

\section{Main results}

As we have seen above, computing the sum of element orders of finite abelian groups is reduced to $p$-groups. For such a group $G$ we can determine $\psi(G)$ by using Corollary 4.4 of [6].

Theorem 1. Let $G={\underset{i=1}{k}}_{\mathbb{Z}_{p^{\alpha_{i}}}}$ be a finite abelian p-group, where $1 \leq$ $\alpha_{1} \leq \alpha_{2} \leq \ldots \leq \alpha_{k}$. Then

$$
\psi(G)=1+\sum_{\alpha=1}^{\alpha_{k}}\left(p^{2 \alpha} f_{\left(\alpha_{1}, \alpha_{2}, \ldots, \alpha_{k}\right)}(\alpha)-p^{2 \alpha-1} f_{\left(\alpha_{1}, \alpha_{2}, \ldots, \alpha_{k}\right)}(\alpha-1)\right),
$$

where

$$
f_{\left(\alpha_{1}, \alpha_{2}, \ldots, \alpha_{k}\right)}(\alpha)= \begin{cases}p^{(k-1) \alpha}, & \text { if } 0 \leq \alpha \leq \alpha_{1} \\ p^{(k-2) \alpha+\alpha_{1}}, & \text { if } \alpha_{1} \leq \alpha \leq \alpha_{2} \\ \vdots & \\ p^{\alpha_{1}+\alpha_{2}+\ldots+\alpha_{k-1},}, & \text { if } \alpha_{k-1} \leq \alpha .\end{cases}
$$

Remarks. 1. The function $f_{\left(\alpha_{1}, \alpha_{2}, \ldots, \alpha_{k}\right)}$ in Theorem 1 is increasing. 2. $\psi\left(\underset{i=1}{X_{i}} \mathbb{Z}_{p^{\alpha_{i}}}\right)$ is a polynomial in $p$ of degree $2 \alpha_{k}+\alpha_{k-1}+\ldots+\alpha_{1}$.

3. An alternative way to write $\psi\left({\underset{i=1}{k}}_{\mathbb{Z}_{p^{\alpha_{i}}}}\right)$ is

$$
\psi\left(\underset{i=1}{k} \mathbb{Z}_{p^{\alpha_{i}}}\right)=p^{2 \alpha_{k}+\alpha_{k-1}+\ldots+\alpha_{1}}-(p-1) \sum_{\alpha=0}^{\alpha_{k}-1} p^{2 \alpha} f_{\left(\alpha_{1}, \alpha_{2}, \ldots, \alpha_{k}\right)}(\alpha) .
$$


3 ON THE SUM OF ELEMENT ORDERS OF FINITE ABELIAN GROUPS $\mathbf{3}$

Theorem 1 allows us to obtain a precise expression of $\psi(G)$ for some particular finite abelian $p$-groups $G$.

Corollary 2. We have:

a) $\psi\left(\mathbb{Z}_{p^{n}}\right)=\frac{p^{2 n+1}+1}{p+1}$

b) $\psi\left(\mathbb{Z}_{p}^{n}\right)=p^{n+1}-p+1$;

c) $\psi\left(\mathbb{Z}_{p^{2}} \times \mathbb{Z}_{p}^{n-2}\right)=p^{n+2}-p^{n+1}+p^{n}-p+1$;

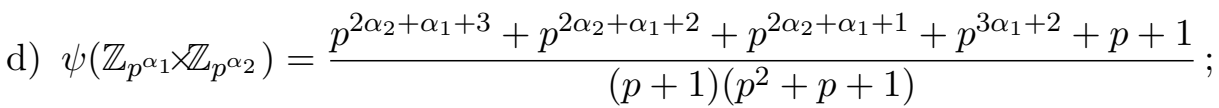

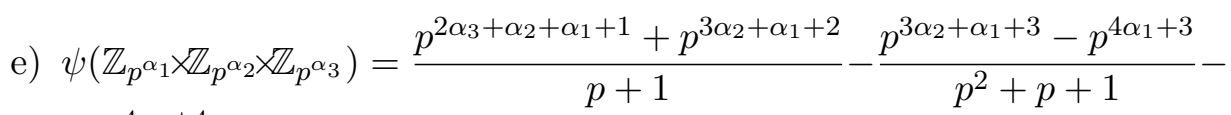
$\frac{p^{4 \alpha_{1}+4}-1}{p^{3}+p^{2}+p+1}$.

Given a positive integer $n$, it is well-known that there is a bijection between the set of types of abelian groups of order $p^{n}$ and the set $P_{n}=$ $\left\{\left(x_{1}, x_{2}, \ldots, x_{n}\right) \in \mathbb{N}^{n} \mid x_{1} \geq x_{2} \geq \ldots \geq x_{n}, x_{1}+x_{2}+\ldots+x_{n}=n\right\}$ of partitions of $n$, namely the map

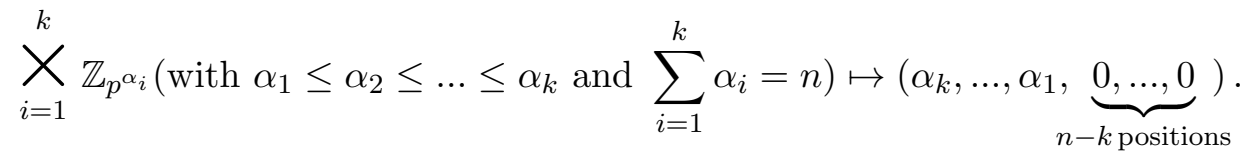

Moreover, recall that $P_{n}$ is totally ordered under the lexicographic order $\preceq$, where

$$
\left(x_{1}, x_{2}, \ldots, x_{n}\right) \prec\left(y_{1}, y_{2}, \ldots, y_{n}\right) \Leftrightarrow\left\{\begin{array}{l}
x_{1}=y_{1}, \ldots, x_{m}=y_{m} \\
\text { and } \\
x_{m+1}<y_{m+1} \text { for some } m \in\{0,1, \ldots, n-1\} .
\end{array}\right.
$$

Obviously, the lexicographic order induces a total order on the set of types of abelian $p$-groups of order $p^{n}$.

By computing the values of $\psi$ corresponding to all types of abelian $p$ groups of order $p^{2}, p^{3}$ and $p^{4}$, respectively, one obtains:

- $\psi\left(\mathbb{Z}_{p}^{2}\right)=p^{3}-p+1<\psi\left(\mathbb{Z}_{p^{2}}\right)=p^{4}-p^{3}+p^{2}-p+1$ 
- $\psi\left(\mathbb{Z}_{p}^{3}\right)=p^{4}-p+1<\psi\left(\mathbb{Z}_{p} \times \mathbb{Z}_{p^{2}}\right)=p^{5}-p^{4}+p^{3}-p+1<\psi\left(\mathbb{Z}_{p^{3}}\right)=$ $p^{6}-p^{5}+p^{4}-p^{3}+p^{2}-p+1$;

- $\psi\left(\mathbb{Z}_{p}^{4}\right)=p^{5}-p+1<\psi\left(\mathbb{Z}_{p} \times \mathbb{Z}_{p} \times \mathbb{Z}_{p^{2}}\right)=p^{6}-p^{5}+p^{4}-p+1<\psi\left(\mathbb{Z}_{p^{2}} \times \mathbb{Z}_{p^{2}}\right)=$ $p^{6}-p^{4}+p^{3}-p+1<\psi\left(\mathbb{Z}_{p} \times \mathbb{Z}_{p^{3}}\right)=p^{7}-p^{6}+p^{5}-p^{4}+p^{3}-p+1<$ $\psi\left(\mathbb{Z}_{p^{4}}\right)=p^{8}-p^{7}+p^{6}-p^{5}+p^{4}-p^{3}+p^{2}-p+1$.

The above inequalities suggest us that the function $\psi$ is strictly increasing. This is true, as shows the following theorem.

Theorem 3. Let $G_{1}={\underset{i=1}{K}}_{\mathbb{Z}_{p^{\alpha_{i}}}}$ and $G_{2}={\underset{j=1}{\mathrm{X}}}_{\mathbb{Z}_{p^{\beta_{j}}}}$ be two finite abelian p-groups of order $p^{n}$. Then

$$
\psi\left(G_{1}\right)<\psi\left(G_{2}\right) \Longleftrightarrow(\alpha_{k}, \ldots, \alpha_{1}, \underbrace{0, \ldots, 0}_{n-k \text { positions }}) \prec(\beta_{r}, \ldots, \beta_{1}, \underbrace{0, \ldots, 0}_{n-r \text { positions }}) .
$$

Proof. First of all, we remark that it suffices to prove $(*)$ only for consecutive partitions of $n$ because $P_{n}$ is fully ordered.

Assume that $\left(\alpha_{k}, \ldots, \alpha_{1}, 0, \ldots, 0\right) \prec\left(\beta_{r}, \ldots, \beta_{1}, 0, \ldots, 0\right)$. We have to prove $\psi\left(G_{1}\right)<\psi\left(G_{2}\right)$ (notice that this inequality holds for the first two elements of $P_{n}$, by b) and c) of Corollary 2). Let $s \in\{1,2, \ldots, r-1\}$ such that $\beta_{1}=\beta_{2}=\cdots=\beta_{s}<\beta_{s+1}$. We distinguish the following two cases.

Case 1. $\beta_{1} \geq 2$

Then $\left(\alpha_{k}, \ldots, \alpha_{1}, 0, \ldots, 0\right)$ is of type $\left(\beta_{r}, \ldots, \beta_{2}, \beta_{1}-1,1,0, \ldots, 0\right)$, i.e. $k=$ $r+1, \alpha_{1}=1, \alpha_{2}=\beta_{1}-1$ and $\alpha_{i}=\beta_{i-1}$ for $i=3,4, \ldots, r+1$. We infer that $f_{\left(\alpha_{1}, \alpha_{2}, \ldots, \alpha_{k}\right)}(\gamma)=f_{\left(\beta_{1}, \beta_{2}, \ldots, \beta_{r}\right)}(\gamma), \forall \gamma \geq \beta_{1}$. One obtains

$$
\begin{aligned}
\psi\left(G_{2}\right)-\psi\left(G_{1}\right) & =p^{\beta_{r}+n}-(p-1) \sum_{\gamma=0}^{\beta_{r}-1} p^{2 \gamma} f_{\left(\beta_{1}, \beta_{2}, \ldots, \beta_{r}\right)}(\gamma) \\
& -p^{\alpha_{k}+n}+(p-1) \sum_{\gamma=0}^{\alpha_{k}-1} p^{2 \gamma} f_{\left(\alpha_{1}, \alpha_{2}, \ldots, \alpha_{k}\right)}(\gamma) \\
& =(p-1) \sum_{\gamma=1}^{\beta_{1}-1} p^{2 \gamma}\left(f_{\left(\alpha_{1}, \alpha_{2}, \ldots, \alpha_{k}\right)}(\gamma)-f_{\left(\beta_{1}, \beta_{2}, \ldots, \beta_{r}\right)}(\gamma)\right) \\
& =(p-1) \sum_{\gamma=1}^{\beta_{1}-1} p^{2 \gamma}\left(p^{(r-1) \gamma+1}-p^{(r-1) \gamma}\right)>0 .
\end{aligned}
$$


Case 2. $\beta_{1}=1$

Then $\left(\alpha_{k}, \ldots, \alpha_{1}, 0, \ldots, 0\right)$ is of type $\left(\beta_{r}, \ldots, \beta_{s+1}-1, \beta_{t}^{\prime}, \beta_{t-1}^{\prime}, \ldots, \beta_{1}^{\prime}, 0, \ldots, 0\right)$, where $\beta_{s+1}-1 \geq \beta_{t}^{\prime} \geq \beta_{t-1}^{\prime} \geq \ldots \geq \beta_{1}^{\prime} \geq 1$ and $\beta_{t}^{\prime}+\beta_{t-1}^{\prime}+\ldots+\beta_{1}^{\prime}=s+1$. We infer that $f_{\left(\alpha_{1}, \alpha_{2}, \ldots, \alpha_{k}\right)}(\gamma)=f_{\left(\beta_{1}, \beta_{2}, \ldots, \beta_{r}\right)}(\gamma), \forall \gamma \geq \beta_{s+1}$. So, we can suppose that $s=r-1$, i.e. $\left(\alpha_{k}, \ldots, \alpha_{1}, 0, \ldots, 0\right)=\left(\beta_{r}-1, \beta_{t}^{\prime}, \beta_{t-1}^{\prime}, \ldots, \beta_{1}^{\prime}, 0, \ldots, 0\right)$. One obtains

$$
\psi\left(G_{2}\right)-\psi\left(G_{1}\right)=p^{\beta_{r}+n}-(p-1) \sum_{\gamma=0}^{\beta_{r}-1} p^{2 \gamma} f_{\left(\beta_{1}, \beta_{2}, \ldots, \beta_{r}\right)}(\gamma)-p^{\beta_{r}+n-1}+S,
$$

where

$$
S=(p-1) \sum_{\gamma=0}^{\beta_{r}-2} p^{2 \gamma} f_{\left(\alpha_{1}, \alpha_{2}, \ldots, \alpha_{k}\right)}(\gamma)>0
$$

Since

$$
f_{\left(\beta_{1}, \beta_{2}, \ldots, \beta_{r}\right)}(\gamma)= \begin{cases}p^{(r-1) \gamma}, & \text { if } 0 \leq \gamma \leq 1 \\ p^{r-1}, & \text { if } 1 \leq \gamma\end{cases}
$$

it follows that

$$
\begin{aligned}
& \psi\left(G_{2}\right)-\psi\left(G_{1}\right)>p^{\beta_{r}+n}-p^{\beta_{r}+n-1}-(p-1) \sum_{\gamma=0}^{\beta_{r}-1} p^{2 \gamma} f_{\left(\beta_{1}, \beta_{2}, \ldots, \beta_{r}\right)}(\gamma) \\
& =p^{\beta_{r}+n}-p^{\beta_{r}+n-1}-(p-1)\left[1+p^{r-1} \frac{p^{2 \beta_{r}}-p^{2}}{p^{2}-1}\right] \\
& =\frac{1}{p+1}\left[p^{\beta_{r}+n-1}\left(p^{2}-p-1\right)+p^{r+1}-p^{2}+1\right]>0 .
\end{aligned}
$$

Conversely, assume that $\psi\left(G_{1}\right)<\psi\left(G_{2}\right)$, but $\left(\alpha_{k}, \ldots, \alpha_{1}, 0, \ldots, 0\right) \succeq$ $\left(\beta_{r}, \ldots, \beta_{1}, 0, \ldots, 0\right)$. Then the first part of the proof leads to $\psi\left(G_{2}\right) \leq \psi\left(G_{1}\right)$, a contradiction. Hence $(*)$ holds.

Two immediate consequences of Theorem 3 are the following.

Corollary 4. Let $n$ be a positive integer and $G$ be an abelian p-group of order $p^{n}$. Then the minimum value of $\psi(G)$ is obtained for $G$ elementary abelian, while the maximum value of $\psi(G)$ is obtained for $G$ cyclic.

Corollary 5. Two finite abelian p-groups of the same order are isomorphic if and only if they have the same sum of element orders. 
Inspired by Corollary 5, we came up with the following conjecture, which we have verified by computer for all abelian groups of order less or equal to 100000.

Conjecture 6. Two finite abelian groups of the same order are isomorphic if and only if they have the same sum of element orders.

In order to decide if two finite abelian groups $G_{1}$ and $G_{2}$ are isomorphic by using the above results, the condition $\left|G_{1}\right|=\left|G_{2}\right|$ is essential, as shows the following simple example.

Example. We have $\mathbb{Z}_{2}^{2} ¥ \mathbb{Z}_{3}$ even if $\psi\left(\mathbb{Z}_{2}^{2}\right)=\psi\left(\mathbb{Z}_{3}\right)=7$.

This proves that the function $\psi$ is not injective, too. The surjectivity of $\psi$ also fails because $\operatorname{Im}(\psi)$ contains only odd positive integers (notice that in fact more can be said, namely: $\psi(G)$ is odd for all finite groups $G$ ). Moreover, there exist odd positive integers not contained in $\operatorname{Im}(\psi)$, as 5 .

Finally, we observe that $\psi(G)$ is not divisible by $|G|$ for large classes of finite groups $G$, as $p$-groups, groups of order $p^{n} q$ ( $p, q$ primes) without normal Sylow $q$-subgroups and groups of even order. More precisely, by MAGMA we checked that there are only three types of groups of order at most 2000 satisfying $|G| \mid \psi(G)$ (the smallest order of such a group $G$ is 105 and $\psi(G)=1785=105 \cdot 17)$ and these are not abelian. Consequently, the study of this property for abelian groups seems to be interesting.

Theorem 7. There are finite abelian groups $G$ such that $\psi(G) \equiv$ $0(\bmod |G|)$.

Proof. Let $G=\mathbb{Z}_{13} \times \mathbb{Z}_{13} \times \mathbb{Z}_{23}$. We have $|G|=3887$ and

$$
\begin{aligned}
\psi(G) & =\psi\left(\mathbb{Z}_{13} \times \mathbb{Z}_{13}\right) \psi\left(\mathbb{Z}_{23}\right)=\left(13^{3}-13+1\right) \frac{23^{3}+1}{23+1} \\
& =1107795=3887 \cdot 285,
\end{aligned}
$$

completing the proof.

We end our note by indicating a natural generalization of $\psi(G)$, which is obtained by replacing the orders of elements with the orders of elements relative to a certain subgroup of $G$.

Open problem. Let $G$ be a finite group. For every subgroup $H$ of $G$, we define the function

$$
\psi_{H}(G)=\sum_{a \in G} o_{H}(a)
$$


where $o_{H}(a)$ denotes the order of $a \in G$ relative to $H$ (that is, the smallest positive integer $m$ such that $\left.a^{m} \in H\right)$. Study the connections between $\psi(G)$ and the collection $\left(\psi_{H}(G)\right)_{H \leq G}$, as well as the minimum/maximun of $\left\{\psi_{H}(G)|H \leq G| H \mid,=n\right\}$, where $n \in \mathbb{N}^{*}$ is fixed.

Acknowledgements. The authors are grateful to the reviewers for their remarks which improve the previous version of the paper.

\section{REFERENCES}

1. Amiri, H.; Jafarian Amiri, S.M.; IsaAcs, I.M. - Sums of element orders in finite groups, Comm. Algebra, 37 (2009), 2978-2980.

2. Amiri, H.; Jafarian Amiri, S.M. - Sum of element orders on finite groups of the same order, J. Algebra Appl., 10 (2011), 187-190.

3. Huppert, B. - Endliche Gruppen, I, Die Grundlehren der Mathematischen Wissenschaften, Band, 134, Springer-Verlag, Berlin-New York, 1967.

4. IsaAcs, I. MARTin - Finite Group Theory, Graduate Studies in Mathematics, 92, American Mathematical Society, Providence, RI, 2008.

5. Schmidt, R. - Subgroup Lattices of Groups, de Gruyter Expositions in Mathematics, 14, Walter de Gruyter \& Co., Berlin, 1994.

6. TĂRnăucEAnu, M. - An arithmetic method of counting the subgroups of a finite abelian group, Bull. Math. Soc. Sci. Math. Roumanie (N.S.), 53 (2010), 373-386.

Received: 21.IX.2011

Revised: 7.XII.2011

Accepted: 10.XII.2011
Faculty of Mathematics, "Al.I. Cuza" University,

Iaşi,

ROMANIA

tarnauc@uaic.ro

dan.ms.chaos@gmail.com 